\title{
Dielectrophoretic tweezers using sharp probe electrode
}

\author{
Kiha Lee, Soon Geun Kwon, Soo Hyun Kim*, Yoon Keun Kwak \\ Department of Mechanical Engineering, Korea Advanced Institute of Science and Technology, Republic of Korea \\ Received 21 December 2004; received in revised form 12 October 2006; accepted 21 October 2006 \\ Available online 1 December 2006
}

\begin{abstract}
The pick and place ability, precise positioning and high spatial resolution of end-effector can be defined as the functional requirements of dielectrophoretic tweezers. The tweezers can manipulate objects in any direction with the strength of dielectrophoretic force. A localized and 3D movable electric field configuration is proposed here and analyzed for the functional requirements of dielectrophoretic tweezers. To achieve a steeply focused field, an electrochemical machining method was developed for a sharp probe electrode, and the developed dielectrophoretic tweezers was used to manipulate cells and beads.
\end{abstract}

(C) 2006 Elsevier B.V. All rights reserved.

Keywords: Dielectrophoresis; Tweezers; Electrochemical machining

\section{Introduction}

Manipulating single cells for operating various functions and probing characteristics of cell is important in biotechnology. Two major approaches have been developed to manipulate individual biological objects. First, a mechanical approach that uses instruments such as miniaturized tweezers and scanning probe microscopes [1,2]. Second, a trapping approach that uses the interaction between a field and an object, such as optical tweezers and dielectrophoretic (DEP) trapping [3,4].

DEP manipulation has advantages over other methods. First, the magnitude and direction of a force can be manipulated by controlling the voltage frequency. It enables to pick and place the micro/nano particle with a simple control of DEP voltage. Secondly, various field configurations with a high gradient can be designed and performed with the aid of microelectrodes. The high gradient of electric field guarantees the deterministic motion of the particle by overcoming the random motion such as Brownian motion of the particle in fluid environment. These advantages and application environment make dielectrophoresis an important application in biotechnology, particularly in fields such as microfluidic systems [5].

For advanced manipulation, DEP tweezers can be used to actively choose a target from a range of objects and, with its

\footnotetext{
* Corresponding author.

E-mail address: soohyun@kaist.ac.kr (S.H. Kim).
}

pickup and placement ability, can precisely position the target. Microelectrode methods have been used for this purpose; for example a dual disk sealed in a capillary and a single capacitively coupled electrode [6,7]. However, these methods require analysis for the electric field and definition of the functional requirements of DEP tweezers.

Previous works using DEP have used a geometry of 2D microelectrode. They have limitations on the movement and placement of a particle at a desired position. The methods using 2D microelectrodes have shown results mainly on trapping of multiple particles. In general, the size of 2D microelectrode is larger than the objects such as a single bead or cell, which causes the difficulty in manipulation for a single particle.

In this paper, for the functional requirement of the tweezer such as pickup, positioning and placement, a probe-type tweezer is fabricated using electrochemical etching. For a single particle manipulation, an ultra sharp electrode with the range of 200-300 nm in its radius of curvature (ROC) was chosen for the tweezer. The performance of picking and placement of a single bead or cell using dielectrophoretic manipulation is demonstrated.

\section{System configuration}

When a polarizable object is affected by an external electric field, the relationship between the induced dipole $(p)$ and the 
electric field $(E)$ can be expressed as follows:

$p=\alpha E$

where $\alpha$ is the relative polarizability of the object to the medium.

The resultant force $(F)$ of the interaction between the electric field and the induced dipole can be expressed as follows:

$F=-\nabla U=-\nabla(-p \cdot E)=\alpha \nabla E^{2}$

where $U$ is the potential energy and $\nabla E^{2}$ means the nonuniformity of the field that also determines the direction and magnitude of the force [3].

In DEP case, the DEP force $\left(F_{\mathrm{DEP}}\right)$ can be expressed as follows:

$F_{\mathrm{DEP}}=2 \pi r^{3} \varepsilon_{\mathrm{m}} \operatorname{Re}\left(\frac{\varepsilon_{\mathrm{o}}^{*}-\varepsilon_{\mathrm{m}}^{*}}{\varepsilon_{\mathrm{o}}^{*}+2 \varepsilon_{\mathrm{m}}^{*}}\right) \nabla E^{2}, \quad \varepsilon^{*}=\varepsilon-j \frac{\sigma}{\omega}$

where $\varepsilon_{\mathrm{m}}$ is the medium permittivity, and $\varepsilon_{\mathrm{o}}^{*}$ and $\varepsilon_{\mathrm{m}}^{*}$ are the complex permittivity of the object and the medium. The complex permittivity defined in Eq. (3) is composed of the permittivity $(\varepsilon)$, the conductivity $(\sigma)$ and the voltage frequency $(\omega)$. Because $\operatorname{Re}\left(\left(\varepsilon_{\mathrm{o}}^{*}-\varepsilon_{\mathrm{m}}^{*}\right) /\left(\varepsilon_{\mathrm{o}}^{*}+2 \varepsilon_{\mathrm{m}}^{*}\right)\right)$ is a frequency dependent parameter, the DEP force to objects in the medium can be changed by applying a varied temporal electric field.

Fig. 1(a) shows the proposed electrode configuration, which consists of a probe and planar electrodes, which also defines the probe angle $(\theta)$, the probe diameter $(d)$ and the electrode distance $(h)$. Fig. 1(b) shows the electric field. The arrow refers to $E$ and the contour indicates the gradient of the electric field square $\left(\nabla E^{2}\right)$ that is proportional to the DEP force. The convergent DEP force, which is formed near the probe-end, can be defined as the end-effector of the DEP tweezers. In a

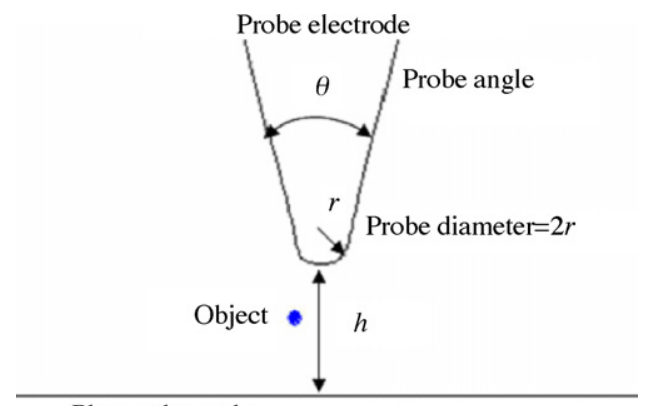

Planar electrode

(a)

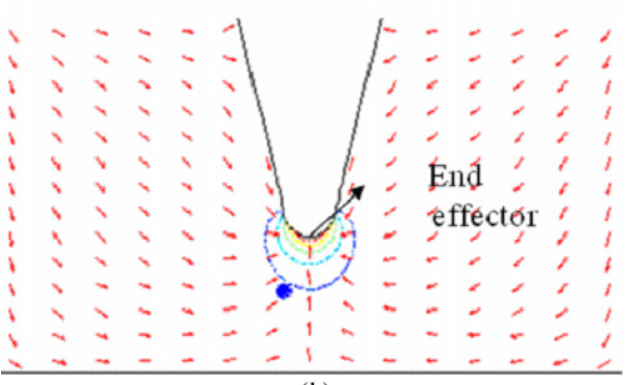

(b)

Fig. 1. Configuration of the probe-planar electrodes: (a) schematic diagram of proposed probe-planar electrode geometry and (b) direction of dielectrophoretic force applied on the particle.
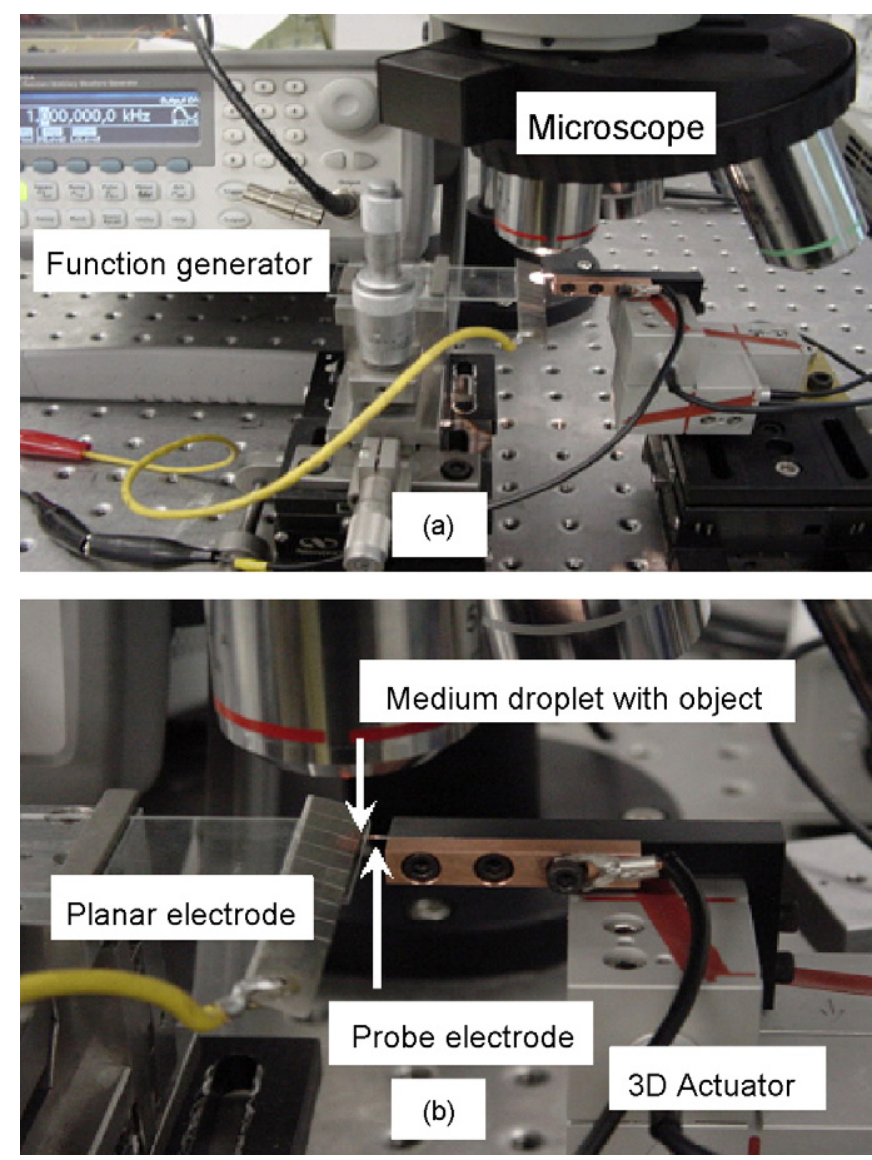

Fig. 2. The experimental setup: (a) experimental apparatus integrated with the inverted microscope and (b) the components of the experimental setup; knife edge as a planar electrode, tungsten electrode as a probe electrode, and 3D piezo actuator for linear 3D actuation.

positive DEP case, the object within the spatial resolution of the probe-end moves to the end-effector and this phenomenon can be defined as a pick process. In a negative DEP case, the object moves away from the end-effector, and this phenomenon can be defined as a place process. Because this configuration is inherently unstable in a positive DEP case, we achieved stable trapping of the end-effector by imposing radial stabilization and by using the boundary of the probe-end for vertical stabilization, which means that the vertical motion of a object is blocked due to contact at the electrode boundary [8].

Fig. 2 shows the experimental setup. The actuating system places the end-effector at a desired position, while the vision system senses the motion. The function generator controls the magnitude and frequency of the electric field between the electrodes, and the resultant concentric and 3D movable DEP force distribution is defined as the DEP tweezers.

\section{Simulation and analysis}

The spatial resolution and force magnitude of the DEP tweezers are critical factors for deterministic, individual manipulation. We therefore defined these critical factors as objective functions and analyzed their performance in relation to the 

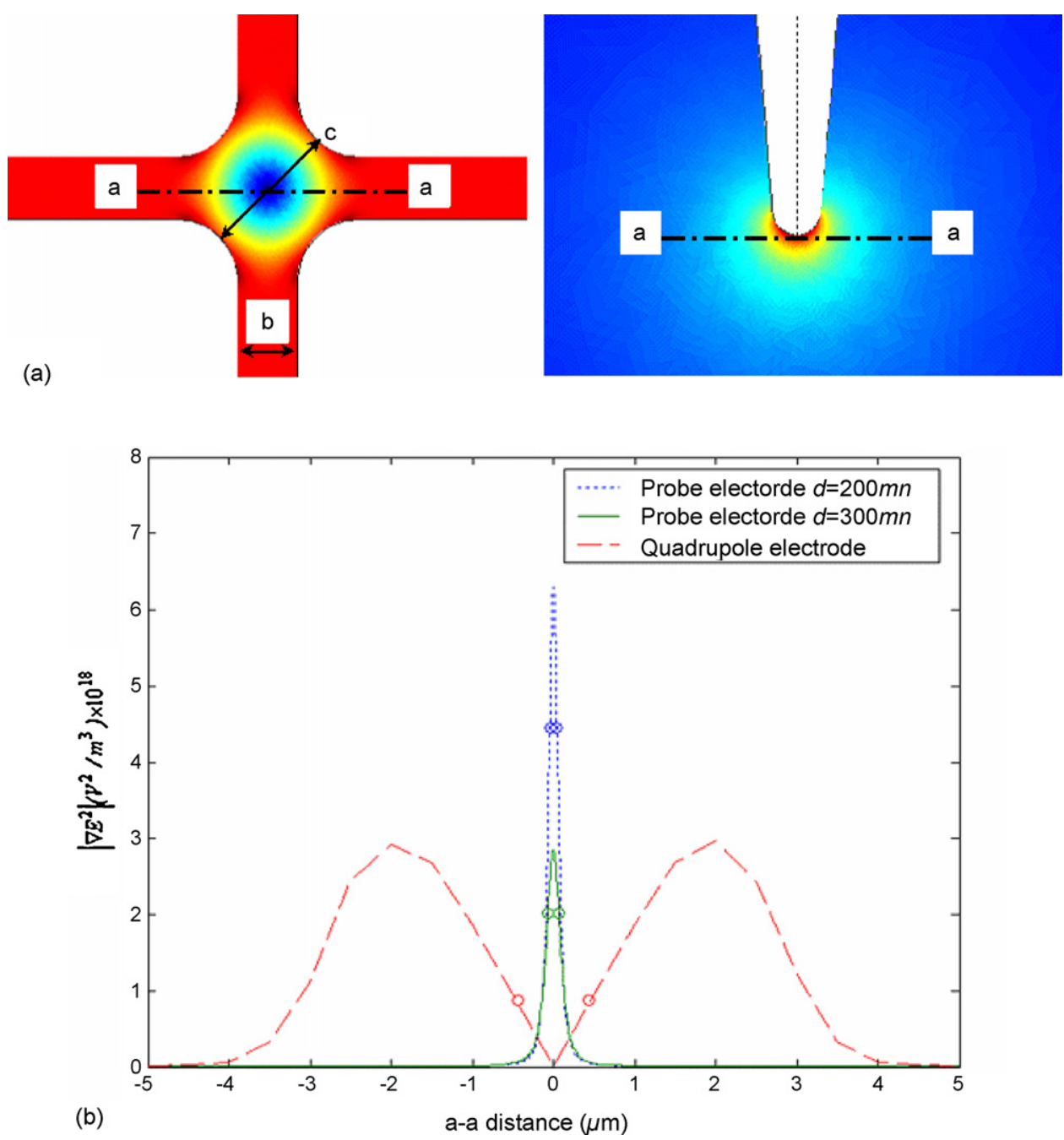

Fig. 3. Comparison of the quadrupole and probe electrodes: (a) the configuration of the quadrupole and probe electrodes and (b) $\nabla E^{2}$ distribution of the quadrupole and probe electrodes.

design parameters, which were defined as $\theta$ and $d$. Furthermore, the spatial resolution of DEP tweezers is set near $3 \mathrm{~dB}$ point from the maximum DEP force, and the analysis results are obtained using a $2 \mathrm{D}$ finite element method (FEMLAB, COMSOL Inc., USA).

Fig. 3(a) shows the configuration of a quadrupole, where the distances $b$ and $c$ are set to $2 \mu \mathrm{m}$ and $6 \mu \mathrm{m}$, respectively, and of the probe electrodes, where $\theta$ and $h$ are set to $10^{\circ}$ and $500 \mu \mathrm{m}$, respectively [9]. The applied voltage was assumed to be $5 \mathrm{~V}_{\mathrm{pp}}$. Fig. 3(b) shows the $\nabla E^{2}$ distribution at the a-a section of the quadrupole and the probe electrodes $(d=200$ and $300 \mathrm{~nm})$. As Eq. (2) indicates, the bottom of the potential well where the objects are trapped, or the point where the maximum DEP force acts, is the center of the $\nabla E^{2}$ distribution if $\operatorname{Re}\left(\left(\varepsilon_{\mathrm{o}}^{*}-\varepsilon_{\mathrm{m}}^{*}\right) /\left(\varepsilon_{\mathrm{o}}^{*}+\right.\right.$ $\left.2 \varepsilon_{\mathrm{m}}^{*}\right)$ )is negative in the quadrupole electrode and positive in the probe electrode. Furthermore, even though there is a magnitude similarity between the maximum $\nabla E^{2}$ of the probe electrode of which diameter is $300 \mathrm{~nm}$ and that of the quadrupole, the spatial resolution can be improved about six times. This represents that the spatial resolution and maximum DEP force can be improved in probe electrode.
Fig. 4(a) and (b) shows that the $\nabla E^{2}$ and spatial resolution can be increased as $d$ or $\theta$ decreases. In objective functions, the magnitude of the maximum DEP force is more sensitive than the spatial resolution over the variation of design parameters; and, in the design parameters, $d$ has the bigger effect than $\theta$. In conclusion, the high $\nabla E^{2}$ value guarantees a high DEP force magnitude when the voltage is low, thereby preventing a medium flow due to Joule heating. Moreover, a high spatial resolution is needed for selectivity, which means that a single object can be manipulated without affecting other objects.

\section{Fabrication of a sharp probe electrode}

For the probe electrode, a tungsten wire with a diameter of $500 \mu \mathrm{m}$ was chosen because of its high electrical conductivity, a $5 \mathrm{M} \mathrm{KOH}$ of electrolyte was used and an electrochemical etching is used to fabricate an ultra sharp probe. [10].

Eqs. (4) and (5) show that a dissolved volume rate $\mathrm{d} V / \mathrm{d} t$ due to etching is proportional to the input current $(i)$, and the etching rate in a radial direction $\left(D_{\mathrm{v}}\right)$ is also proportional to density of 


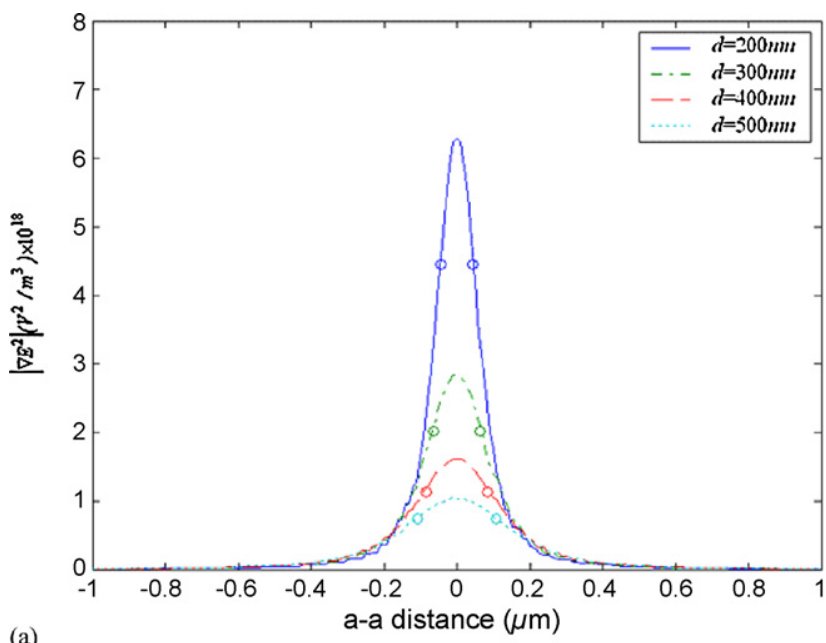

(a)

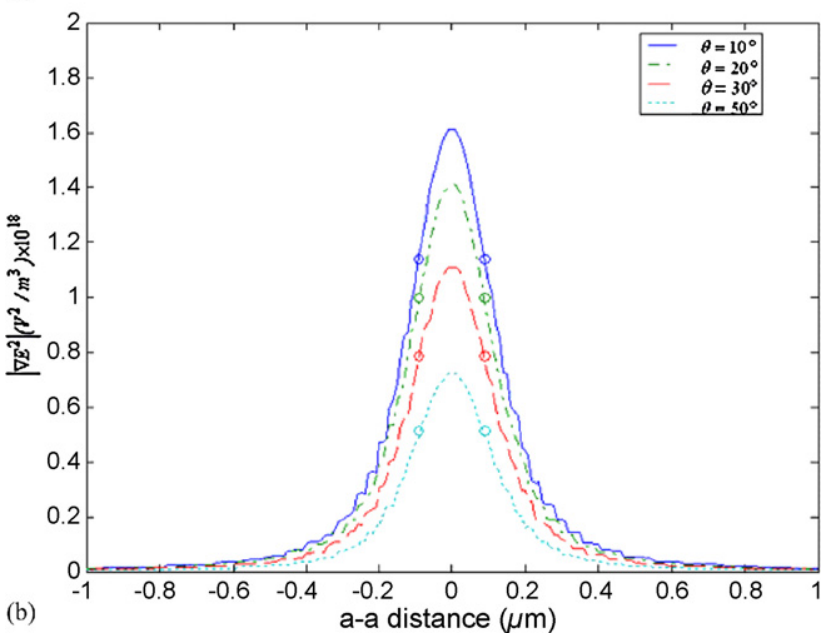

Fig. 4. Objective function distribution according to the design parameters. $\nabla E^{2}$ distribution according to the (a) probe diameter variation and the (b) probe angle variation.

the input current $(J)$, relationships are expressed as follows:

$\frac{\mathrm{d} V}{\mathrm{~d} t}=\frac{A}{\rho_{W} z F} i=\alpha_{\mathrm{e}} i$

$D_{\mathrm{v}}=\frac{\mathrm{d} V}{\mathrm{~d} t} \frac{1}{A_{\mathrm{W}}}=\alpha_{\mathrm{e}} \frac{i}{A_{\mathrm{W}}}=\alpha_{\mathrm{e}} J$

where $V$ is the etched volume, $A$ the atomic weight, $\rho_{\mathrm{W}}$ the density, $z$ the valence, $F$ the Faraday's constant and $A_{\mathrm{W}}$ is the surface area.

Electrochemical process consists of two steps. First, a cylindrical wire is etched being dipped the electrolyte by elaborately controlling an input current density and voltage to fabricate a shape controlled microelectrode. Fig. 5(a) shows the stepwise tungsten wire after the electrochemical etching. The stepwise electrode shape can be determined by extracting the electrode during the etching.

Second is a electropolishing process to increase an electrode sharpness. We etched the electrode in both radial and longitudinal directions with a constant voltage (3-6 V). Fig. 5(b) shows the sharp electrode through electropolishing by controlling an etching period. During the electropolishing, if we control the

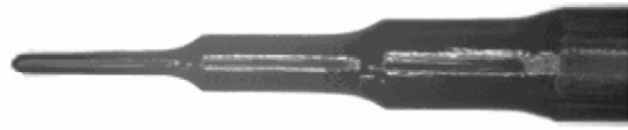

(a)
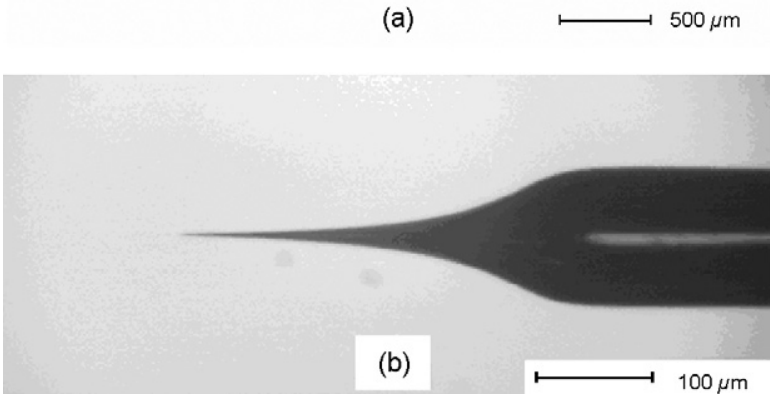

Fig. 5. Fabrication of a sharp probe electrode: (a) fabrication of a stepped uniform electrode using the electrochemical etching and (b) fabrication of a sharp electrode using electropolishing technique.

machining time and immersion depth, we can obtain the various electrode geometries. An electrode with a large $\theta$, such as the one shown in Fig. 6(a), was fabricated with shallow immersion and a long machining time. In contrast, an electrode with a small $\theta$, such as the one shown in Fig. 6(b), was fabricated with deep
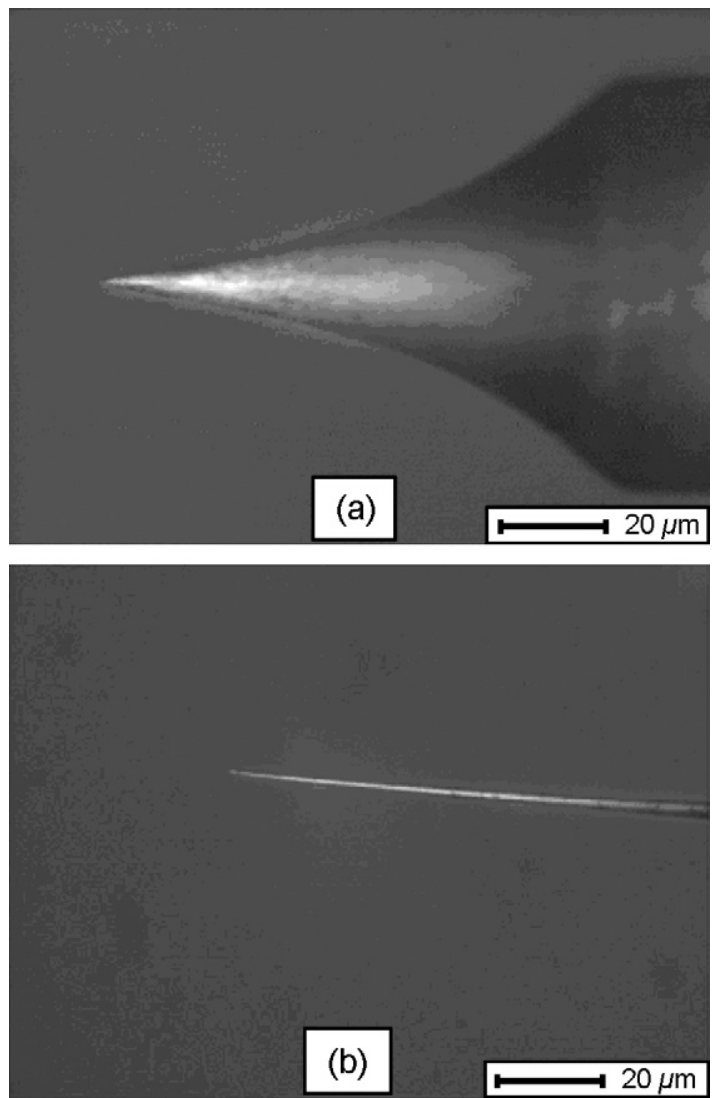

Fig. 6. Fabrication of sharp probes with various aspect ratios by controlling the machining time and depth of immersion. A sharp probe with (a) low aspect ratio and (b) high aspect ratio. 
immersion and a short machining time. This process enabled us to fabricate a sharp electrode with a minimum of $200 \mathrm{~nm}$ in the $d$ and various $\theta$ values.

\section{Voltage control}

The DEP velocity $\left(u_{\mathrm{o} / \mathrm{m}}\right)$ can be defined as the object velocity relative to the medium. However, if the medium flows in $u_{\mathrm{m}}$, the velocity of the object's motion $\left(u_{\mathrm{O}}\right)$ can be expressed as follows:

$u_{\mathrm{o}}=\left[u_{\mathrm{o} / \mathrm{m}}\right]^{\mathrm{DEP}}+\left[u_{\mathrm{m}}\right]^{\mathrm{EHD}}$

where the bracket means the main reason for the motion.

For precise DEP manipulation, we need to minimize the electrohydrodynamics (EHD), which means the medium flow caused by the electric field. The EHD is classified as AC electroosmosis, which has a Gaussian-shaped velocity profile in relation to the voltage frequency, and an electrothermal flow that is mainly a function of the magnitude of the voltage [11].

Fig. 7(a) shows a system impedance model [12]. This model consists of the double layer's, which forms between the electrode and the medium, impedance $\left(Z_{\mathrm{d}}=1 / j \omega C_{\mathrm{d}}\right)$, the medium's impedance $\left(Z_{\mathrm{m}}=R_{\mathrm{m}} /\left(1+j \omega R_{\mathrm{m}} C_{\mathrm{m}}\right)\right)$ and the object's impedance $\left(Z_{\mathrm{o}}=R_{\mathrm{O}} /\left(1+j \omega R_{\mathrm{O}} C_{\mathrm{o}}\right)\right)$.

Fig. 7(b) shows how the $\operatorname{Re}\left(\left(\varepsilon_{\mathrm{o}}^{*}-\varepsilon_{\mathrm{m}}^{*}\right) /\left(\varepsilon_{\mathrm{o}}^{*}+2 \varepsilon_{\mathrm{m}}^{*}\right)\right)$ distribution of a polystyrene object $\left(\varepsilon_{\mathrm{p}}=2 \times 10^{-11} \mathrm{~F} / \mathrm{m}\right.$, $\left.\sigma_{\mathrm{p}}=2.2 \times 10^{-2} \mathrm{~S} / \mathrm{m}\right)$ in a water medium $\left(\varepsilon_{\mathrm{m}}=7 \times 10^{-10} \mathrm{~F} / \mathrm{m}\right.$, $\sigma_{\mathrm{m}}=2 \times 10^{-3} \mathrm{~S} / \mathrm{m}$ ) relates to the voltage frequency.

The AC electroosmosis can be explained by the interaction of the impedances of the double layer and the medium. Fig. 7(c) indicates the main impedance of region $c$ in Fig. 7(b), where the positive DEP force acts, and Fig. 7(d) indicates the main impedance of region $d$ in Fig. 7(b), where the negative DEP force acts. Because the medium's impedance has little effect on these regions, it is possible to implement the pick and place process while minimizing the $\mathrm{AC}$ electroosmosis.
Compared to AC electroosmosis, the electrothermal flow, which owes to Joule heat in conductive medium, is mainly a function of the magnitude of the voltage. Therefore, to perform DEP manipulation while minimizing the effect of the electrothermal flow, we need to maintain $\nabla E^{2}$ while minimizing the $V^{2}$. This result can be achieved by fabricating a sharp electrode that also increases the spatial resolution of the DEP tweezers.

\section{Experimental results}

The distance between the probe electrode $\left(\theta=10^{\circ}\right.$, $d=300 \mathrm{~nm}$ ) and the planar electrode was set to $500 \mu \mathrm{m}$ in our experiments. The position of objects near the probe was affected by the medium's viscosity and probe's motion, this effect was reduced by controlling the actuation speed and by using a high aspect ratio for the probe.

Fig. 8 shows the manipulation of a single yeast cell. Fig. 8(a) and (b) shows the pick process with the aid of a positive DEP in conditions of $50 \mathrm{~Hz}$ and $2 \mathrm{~V}_{\mathrm{pp}}$. The yeast cell near the endeffector was picked up by the DEP tweezers. Fig. 8(c) shows the positioning of a captured single cell by the actuating system. Although the external flow occurred, the cell could not escape from the end-effector. Fig. 8(d) shows the place process with the aid of a negative DEP in conditions of $20 \mathrm{MHz}$ and $3 \mathrm{~V}_{\mathrm{pp}}$. For precise placement, the magnitude of the voltage should be just large enough to detach the target object from the end-effector without disrupting other objects.

Fig. 9(a) and (b) shows the pick process of a $1 \mu \mathrm{m}$ polystyrene bead in a water medium. As the size of the object decreases, the Brownian motion increases. We chose the $30 \mathrm{~Hz}$ and $0.7 \mathrm{~V}_{\mathrm{pp}}$ condition for positive DEP manipulation. In addition to the geometry of the electrode, the magnitude of the voltage is closely related to the spatial resolution of the DEP tweezers. If the magnitude of the voltage is too high, objects around the broadened field move and adhere to the end-effector due to the

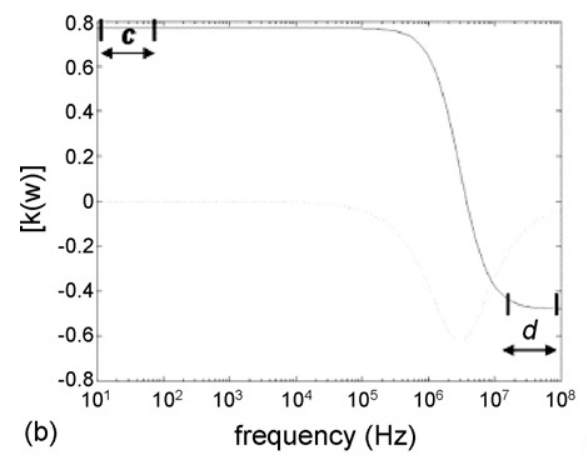

(a)
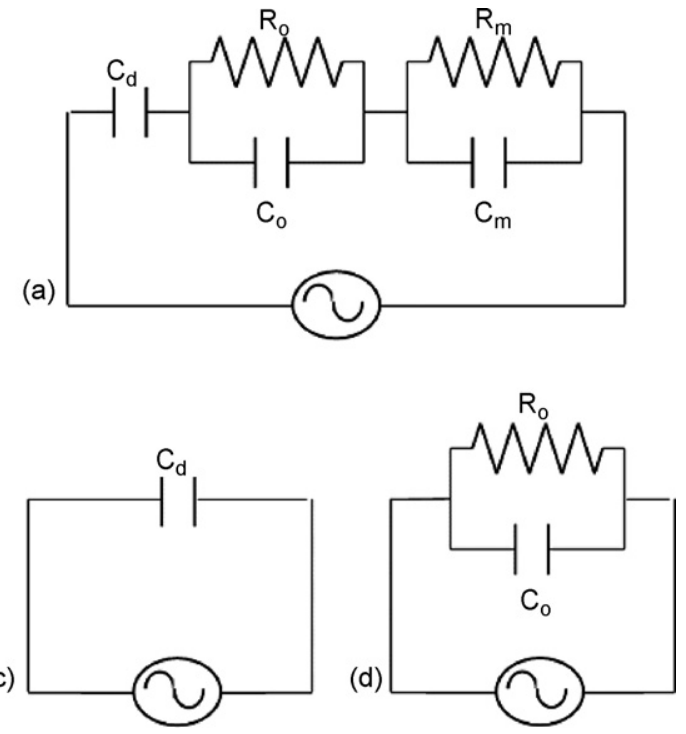

Fig. 7. The system impedance model: (a) impedance model of the system considering the effect of the double layer, object and medium fluid, (b) Clausius-Mossoti factor of polystyrene object in water medium, and ( $\mathrm{c}$ and $\mathrm{d}$ ) a simplified impedance model of the system. 


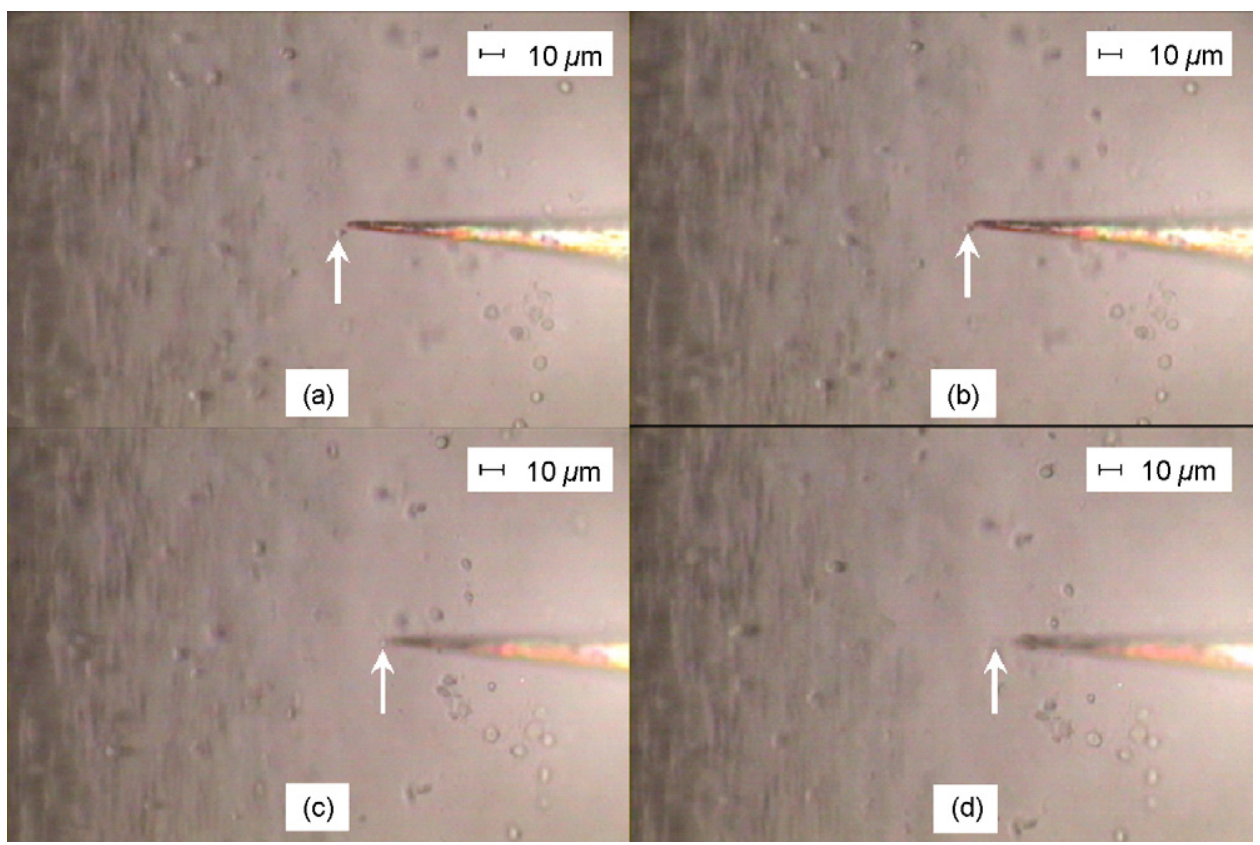

Fig. 8. The manipulation of a single yeast cell in water medium: (a and b) a pick process using positive DEP in conditions of $50 \mathrm{~Hz}$ and $2 \mathrm{~V}_{\mathrm{pp}}$, (c) positioning of the cell trapped at the end of the probe and (d) a placement process using negative DEP in conditions of $20 \mathrm{MHz}$ and $3 \mathrm{~V}_{\mathrm{pp}}$.

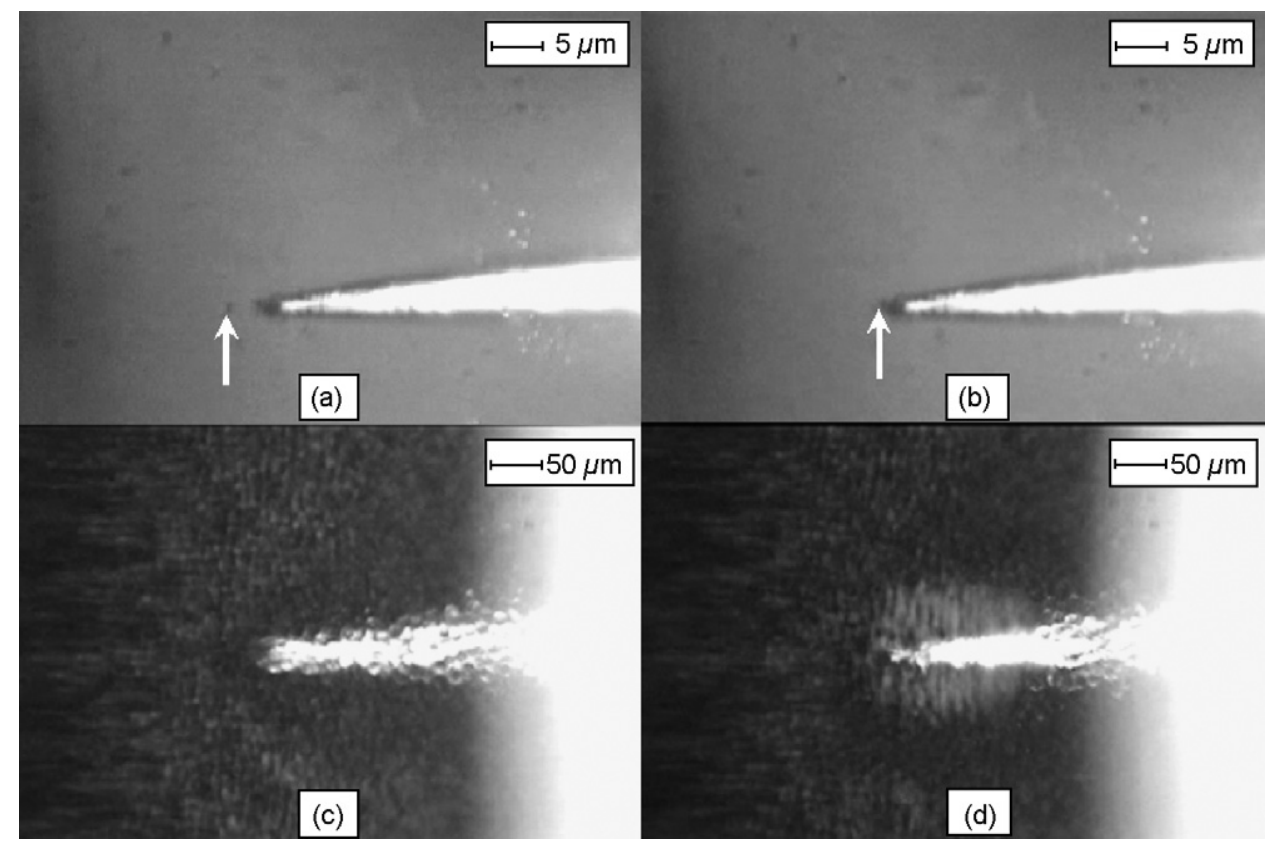

Fig. 9. The manipulation of a polystyrene bead and erythrocyte cells in water medium: (a and b) positive DEP manipulation of a $1 \mu \mathrm{m}$ polystyrene bead in a $30 \mathrm{~Hz}$, $0.7 \mathrm{~V}_{\mathrm{pp}}$ condition, and (c and d) negative DEP manipulation of $10 \mu \mathrm{m}$ erythrocyte cells in a $20 \mathrm{MHz}$ and $10 \mathrm{~V}_{\mathrm{pp}}$ condition.

electrochemical processing between the polystyrene bead and the tungsten probe. This adhesion interrupts the place process. If the magnitude of the voltage is too low, the object escapes from the trapping due to Brownian motion.

Fig. 9(c) and (d) shows the negative DEP manipulation of $10 \mu \mathrm{m}$ erythrocyte cells in a $20 \mathrm{MHz}$ and $10 \mathrm{~V}_{\mathrm{pp}}$ condition. This group handling, which can be applied to a DEP filter for sorting different kinds of cell groups, is a prerequisite for an individual manipulation.

\section{Conclusion}

A 3D movable and concentric electric field for DEP tweezers has been designed to pick and place an individual object with the aid of a frequency dependent property, and the characteristics of the electric field are compared with conventional DEP trapping by investigating the objective functions such as spatial resolution and force magnitude of DEP tweezer. In order to improve the DEP performance, we have used a sharp probe electrode as a test 
bed. By using electrochemical machining, we have fabricated a sharp electrode, which has the size of its end comparable to the single object such as microbead and cell.

For the deterministic manipulation, the voltage was controlled in terms of magnitude and frequency minimizing the effects of electrohydrodynamic flow.

Future work includes the simulation of multiphysics phenomena such as the medium's flow and the thermal problem, and the development of surface modification that prevents electrochemical adhesion. Finally, we hope to configure the electrode for more concentrated electric field and advanced manipulative capabilities, such as rotation.

In short, by using the field gradient trap of a single cell in biotechnology, we have presented an alternative approach for DEP manipulation.

\section{References}

[1] K. Mølhave, T.M. Hansen, D.N. Madsen, P. Bøggild, Towards pick-andplace manipulation of nanostructures, J. Nanosci. Nanotechnol. (2004) 279-282.

[2] G. Binnig, H. Rohrer, Scanning tunneling microscopy, Surf. Sci. (1985) $17-26$.

[3] K.I. McDonald, Laser tweezers, Am. J. Phys. (2000) 486-488.

[4] H.A. Pohl, Dielectrophoresis, Cambridge University Press, 1978.

[5] Hughes, P. Michael, Strategies for dielectrophoretic separation in laboratory-on-a-chip systems, Electrophoresis (2002) 2569-2582.

[6] S. Ogata, T. Yasukawa, T. Matsue, Dielectrophoretic manipulation of a single chlorella cell with dual-microdisk electrode, Bioelectrochemistry (2001) 33-37.

[7] T. Mueller, G. Gradl, S. Howitz, S. Shirley, T. Schnelle, G. Fuhr, A 3D microelectrode system for handling and caging single cells and particles, Biosens. Bioelectron. (1999) 247-256.
[8] T.N. Tombs, T.B. Jones, Effect of moisture on the dielectrophoretic spectra of glass spheres, IEEE Trans. Ind. Appl. (1993) 281-285.

[9] N.G. Green, H. Morgan, Dielectrophoretic investigations of submicrometre latex spheres, J. Phys. D (1997) 2626-2633.

[10] D.I. Kim, H.S. Ahn, Etching voltage control technique for electrochemical fabrication of scanning probe microscope tips, Rev. Sci. Instrum. (2002) 1337-1339.

[11] N.G. Green, A. Ramos, A. Gonzalez, H. Morgan, A. Castellanos, Fluid flow induced by non-uniform ac electric fields in electrolytes on microelectrodes. I. Experimental measurements, Phys. Rev. E (2000) 40114018.

[12] T.B. Jones, M. Gunji, M. Washizu, M.J. Feldman, Dielectrophoretic liquid actuation and nanodroplet formation, J. Appl. Phys. (2001) 1441-1448.

\section{Biographies}

Kiha Lee is a PhD candidate in the Department of Mechanical Engineering, KAIST. He received his BE and MPhil in Mechanical Engineering from KAIST in 1993 and 1996, respectively. He worked as a researcher at LGIS and Mirae in Korea from 1996 to 2003. He is currently working on nano, bio application using dielectrophoresis.

Soon Geun Kwon is a PhD candidate in the Department of Mechanical Engineering, KAIST. He received his BE and MPhil in Mechanical Engineering from KAIST in 2002 and 2004, respectively. He is currently working on Electrochemical fabrication of multi microelectrodes.

Soo Hyun Kim received his BS and MPhil in Mechanical Engineering from Seoul National University in 1978 and KAIST in 1980, respectively. He obtained his $\mathrm{PhD}$ from the Imperial College, University of London in 1991. He is now Professor of Mechanical Engineering with KAIST.

Yoon Keun Kwak received his BS and MPhil in Mechanical Engineering from Seoul National University in 1971 and University of Colorado, Boulder, CO in 1974, respectively. He obtained his PhD from the University of Texas (Austin, TX, USA) in 1978. He is now a Professor of Mechanical Engineering with KAIST. 